\title{
Creation of vectorial bottle-hollow beam using radially or azimuthally polarized light
}

\author{
Huapeng Ye, ${ }^{1}$ Chao Wan, ${ }^{1}$ Kun Huang, ${ }^{1}$ Tiancheng Han, ${ }^{1}$ Jinghua Teng, ${ }^{2}$ Yeo Swee Ping, ${ }^{1}$ and Cheng-Wei Qiu${ }^{1, *}$ \\ ${ }^{1}$ Department of Electrical and Computer Engineering, National University of Singapore, 4 Engineering Drive 3, Singapore 117576 \\ ${ }^{2}$ Institute of Materials Research and Engineering, Agency for Science, Technology and Research (A*STAR), 3 Research Link, Singapore 117602 \\ *Corresponding author: eleqc@nus.edu.sg
}

Received September 30, 2013; revised December 20, 2013; accepted December 21, 2013;

posted December 24, 2013 (Doc. ID 198639); published January 28, 2014

\begin{abstract}
We propose a single-beam generation scheme to obtain a bottle-hollow (BH) beam using a binary phase mask and a focusing lens. The resulting BH beam is shown to possess an open bottle-shaped null intensity region, which has two hollow tube-shaped null intensity regions located on two opposite sides of this bottle. It is found that this scheme works identically under incident illumination with radial or azimuthal polarization. Another advantage of this scheme is that the same binary mask can be employed as a focusing lens with different choices of numerical aperture (NA). Furthermore, we observe that the length of the $\mathrm{BH}$ beam is inversely proportional to $\mathrm{NA}^{2}$ while the diameters of both the bottle and hollow regions are inversely proportional to NA; thereby leading to an adjustable $\mathrm{BH}$ beam. This $\mathrm{BH}$ beam may find attractive applications in noninvasive manipulation of microscopic particles over large distances. (C) 2014 Optical Society of America

OCIS codes: (050.1380) Binary optics; (260.5430) Polarization; (050.1965) Diffractive lenses; (350.4855) Optical tweezers or optical manipulation.

http://dx.doi.org/10.1364/OL.39.000630
\end{abstract}

Using a single light beam for optical trapping [1-ㅗ] and manipulation of micron-sized light-absorbing particles [3-5] has recently attracted the attention of researchers. It has been shown that a single beam with hollow or bottle intensity distribution can be readily utilized for stable manipulation. The high-intensity barrier of the hollow or bottle beam may serve as a repelling 'pipe wall' on particles trapped in the dark region on the axis while the axial component of the thermal photophoretic force pushes particles along the channel [4]. This mechanism minimizes the optical damage due to heating of captured particles at high optical intensity [6]. Optical hollow beams are beams with low (or zero) central intensity channels surrounded by ring-shaped regions with higher intensity, which can be generated using an axicon $[\underline{7}, \underline{8}]$, spatial light modulator $[9,10]$, or holographic phase plates $[\underline{4}, 11]$; whereas, optical bottle beams are beams with low (or zero) intensity bottle-shaped regions surrounded by three-dimensional regions of higher intensity, which can be generated using moiré techniques [2], speckle pattern [1]], spatial light modulator []] , diffractive optical elements [12], axicon [13] , or computer-generated hologram [14].

An optical bottle or hollow beam is usually timeindependent and can be generated via different mechanisms [ $7-14]$. However, it has recently been reported that an optical bottle beam can be dynamic along the transverse direction [15]. Optical bottle and hollow beams are also reported to be generated individually in the same system [13], but in this mechanism they are separated by the objective. Consequently, either hollow beam or bottle beam can only be adopted in trapping and manipulation of particles, and individual properties cannot be integrated into one beam. Functioning similarly to the optofluidic channel in the slot waveguide [16], the optical hollow beam finds applications in transporting particles [1]. However, unlike the optical bottle beam, which acts like a highly volumetric container with appropriate confinement [14], the optical hollow beam cannot be used in manipulating a large amount of particles even though they are precisely delivered to the desired location. The particles transported to the tail of the hollow will lose control, as the field at the tail of the hollow is a diffracted field, without good confinement. Hence, to increase the flexibility of a hollow or bottle beam for optical manipulation, it is highly desirable to generate the bottle-hollow $(\mathrm{BH})$ beam via one system, to combine individual merits. To achieve this aim, we propose a scheme for generating a BH beam using a specially designed binary phase mask and a focusing lens $[\underline{17}, \underline{18}]$. We have observed that both radially and azimuthally polarized illuminations can give rise to a $\mathrm{BH}$ beam in the focal region. The generated $\mathrm{BH}$ beam has an open bottle-shaped null intensity region surrounded by a high-intensity light barrier, and a hollow located at the neck and bottom of the bottle, respectively. More interestingly, we have found during the course of our research that the same binary mask may be employed for lenses with different numerical apertures (NAs), resulting in a $\mathrm{BH}$ beam with various parameters. To the best of our knowledge, the relationship between lens $\mathrm{NA}$ and $\mathrm{BH}$ beam properties, such as length and diameter has not been explicitly studied previously. Our results show that the BH beam length is inversely proportional to $\mathrm{NA}^{2}$ while the diameter of both the bottle and hollow beams is inversely proportional to NA, thus leading to an adjustable $\mathrm{BH}$ beam. This BH beam may find applications in manipulating micron-sized particles over large distances [4].

In principle, the focusing of a vector beam modulated by binary elements can be numerically approximated by Richards and Wolf's theory [19]. Assuming that the incident electric field of the vector beam at the pupil plane in cylindrical coordinate is given by

$$
\vec{E}_{i}(\rho, \phi)=l_{0} P(\rho)\left[\xi(\rho, \phi) \vec{e}_{\rho}+\eta(\rho, \phi) \vec{e}_{\phi}\right],
$$

where $l_{0}$ is the peak field amplitude at the pupil plane, the amplitude $P(\rho)$ at the pupil plane obeys the BesselGaussian distribution [20], and $\xi(\rho, \phi)$ and $\eta(\rho, \phi)$ are 
the field strength factors of the respective components (with both of them satisfying $\xi^{2}+\eta^{2}=1$ ). The incident light is radially polarized when $\xi=1$ and $\eta=0$, or azimuthally polarized when $\xi=0$ and $\eta=1$. From Richards and Wolf's theory [18], the electric field in the focal region can be expressed as

$$
\begin{aligned}
E\left(r, \phi^{\prime}, z\right)= & \frac{-i l_{0} f}{\lambda} \int_{0}^{\theta_{\max }} \int_{0}^{2 \pi} \sin \theta \cdot P(\theta) \cdot \Gamma \\
& \times e^{i k\left(z \cos \theta+r \sin \theta \cos \left(\varphi-\phi^{\prime}\right)\right)} \mathrm{d} \varphi \mathrm{d} \theta,
\end{aligned}
$$

where $P(\theta)$ is the pupil apodization function relative to $P(\rho)[18], k$ is the wave number, and $\Gamma$ is the transmission matrix defined in Eq. (3):

$$
\begin{aligned}
\Gamma= & \xi(f g(\theta), \varphi) \cdot T(\theta) \cdot\left[\begin{array}{c}
\cos \theta \cos \varphi \cdot \vec{e}_{x} \\
\cos \theta \sin \varphi \cdot \vec{e}_{y} \\
\sin \theta \cdot \vec{e}_{z}
\end{array}\right] \\
& +\eta(f g(\theta), \varphi)\left[\begin{array}{c}
-\sin \varphi \cdot \vec{e}_{x} \\
\cos \varphi \cdot \vec{e}_{y} \\
0 \cdot \vec{e}_{z}
\end{array}\right],
\end{aligned}
$$

where $g(\theta)$ is the ray projection function, which is equal to $\sin (\theta)$ for a lens obeying the sine condition, $f$ is the focal length of the objective lens, and $T(\theta)$ denotes the amplitude modulation induced by the binary phase mask. Obviously, Eq. (2) and Eq. (3) are generalized forms of all the field components in the focal region, where the polarization of the illumination is controlled via the appropriate choice of $\xi$ and $\eta$. Figure 1(a) depicts the proposed schematics of the setup to generate the $\mathrm{BH}$ beam. Radially or azimuthally polarized light, which can be produced by modulating linearly polarized laser beam with any of the existing techniques $[\underline{21}, \underline{22}]$, is first collimated and then modulated by the binary phase mask, as portrayed in Fig. 1(b). For convenience, a box labeled as modulator is used to represent the devices that are capable of generating radially or azimuthally polarized light. In this work, we adopt a five-belt silica groove as our phase mask, as depicted in Fig. 1(b). A phase difference of $\pi$ will be created between the bright yellow and dark gray regions. Consequently, the amplitude of the

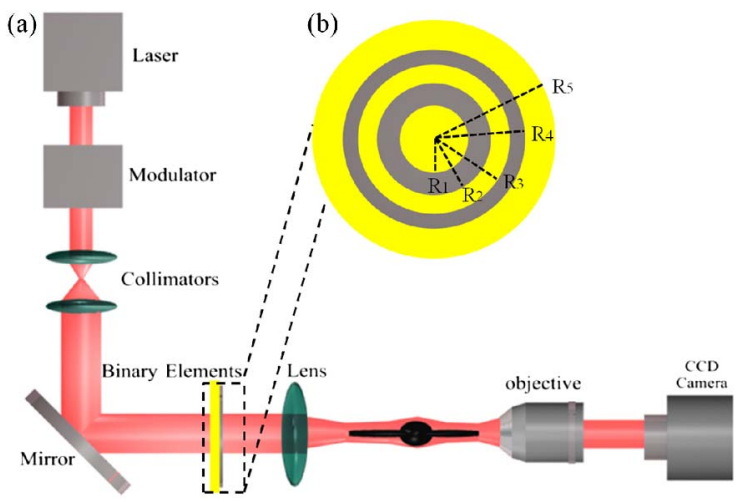

Fig. 1. Schematic setup to generate BH beam with phasecontrolled binary elements. The dark region between the objective and the lens denotes the area where a $\mathrm{BH}$ beam forms. light passing through the phase mask can be expressed as

$$
T(\theta)=\left\{\begin{array}{ll}
1 & \text { for } 0<\theta<\theta_{1}, \theta_{2}<\theta<\theta_{3}, \theta_{4}<\theta<\alpha \\
-1 & \text { for } \theta_{1}<\theta<\theta_{2}, \theta_{3}<\theta<\theta_{4}
\end{array} .\right.
$$

where $\alpha=\arcsin (\mathrm{NA})$ of the focusing lens, and the angle $\theta_{i}(i=1 \sim 4)$ corresponds to the four radial positions $r_{i}=\left(\sin \theta_{i}\right) / \mathrm{NA}$.

To generate the $\mathrm{BH}$ beam, the phase mask is optimized with a particle swarm optimization algorithm [23] to make the constructive interference occur at the high intensity light barrier while destructive interference occurs at the dark region. The optimal parameters of the phase mask are listed in Table 1 . To investigate the beamshaping property of this mask, numerical simulations are performed using Eqs. (1)-(3). Figures 2(a) and 2(b) present the total electric field intensity distributions, when the incident illumination is radially or azimuthally polarized with identical intensity distributions. In both cases, the NA of the objective lens is 0.95. As shown in Fig. 2(a), when the incident light is radially polarized, a closed bottle-shaped distribution is created. In contrast, a $\mathrm{BH}$ beam is produced when the incident light is azimuthally polarized, as shown in Fig. 2(b). It can be inferred from Eqs. (1)-(3) that focused radially polarized beam leads to a vector field with both longitudinal and radial components; whereas, azimuthally polarized incidence generates purely transversal component $(E \varphi)$ in the focal region $[19,24]$. To understand the underlying reason for the distributions in Fig. 2(a), we plot the radial and longitudinal components in Figs. 2(c) and 2(d), both of

Table 1. Optimized Parameters of Binary Phase Mask

\begin{tabular}{lcccc}
\hline$i$ & 1 & 2 & 3 & 4 \\
\hline$\theta$ (degree) & 6.34 & 31.09 & 49.33 & 64.84 \\
\hline
\end{tabular}
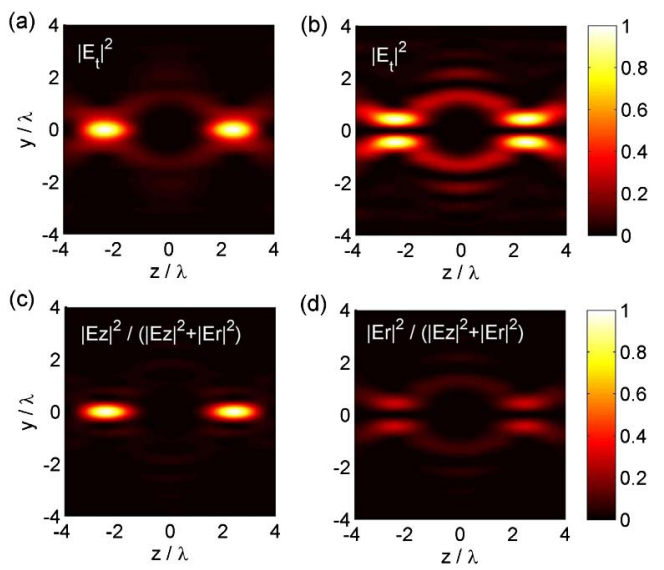

Fig. 2. Generation of $\mathrm{BH}$ beam with a high-NA $(\mathrm{NA}=0.95)$ lens under incident light with (a) radially and (b) azimuthally polarized fields. For radially polarized light, the intensity profiles of its (c) longitudinal and (d) radial components are also shown. 
which are normalized with respect to the total electric field intensity. As can be seen from Fig. 2(c), a strong longitudinal component $(E z)$ arises and two hotspots appear at two separate locations. However, the BHshaped intensity distribution is found in the radial component $(E r)$, as shown in Fig. 2(d). The intensity of the radial component is very weak when compared with its longitudinal counterpart. Thus, we infer that the closed bottle-shaped distribution in Fig. 2(a) originates from the vector property of the total electric field intensity $\left(|E r|^{2}+|E \boldsymbol{z}|^{2}\right)[24]$.

In the case of a low-NA lens, the $\mathrm{BH}$ beam can be generated for both azimuthally and radially polarized incident beams. From Figs. 2(c) and 2(d), it can be observed that the radial component has a BH-shaped distribution while the longitudinal component has two hotspots along the $z$ axis. Therefore, to achieve a $\mathrm{BH}$ beam when the illumination is radially polarized, one has to lower the Ez intensity by adopting a low-NA lens. In this study, NA $\leq 0.1$ is considered as low NA. It is taken as a null field when the energy of longitudinal component at the locations [similar to Fig. 2(c)] where strong longitudinal component is generated is smaller than $1.8 \%$ when NA $\leq 0.1$. Figures 3(a) and 3(b) depict a BH beam which is created when $\overline{\mathrm{NA}}=0.001$ for illumination with radial and azimuthal polarization. As shown in Figs. 3(a) and 3(b), an open bottle-shaped dark region surrounded by a high-intensity barrier is created in the middle, and two hollows are located at the neck and bottom of the bottle, respectively. Obviously, the intensity profiles in Figs. 3(a) and 3(b) are identical to each other due to the fact that the longitudinal component is negligible when focusing radially polarized light with low NA lens. However, the field in Fig. 3(a) is radially polarized, whereas it is azimuthally polarized in Fig. $\underline{3(b)}$. Figure $\underline{3(c)}$ depicts
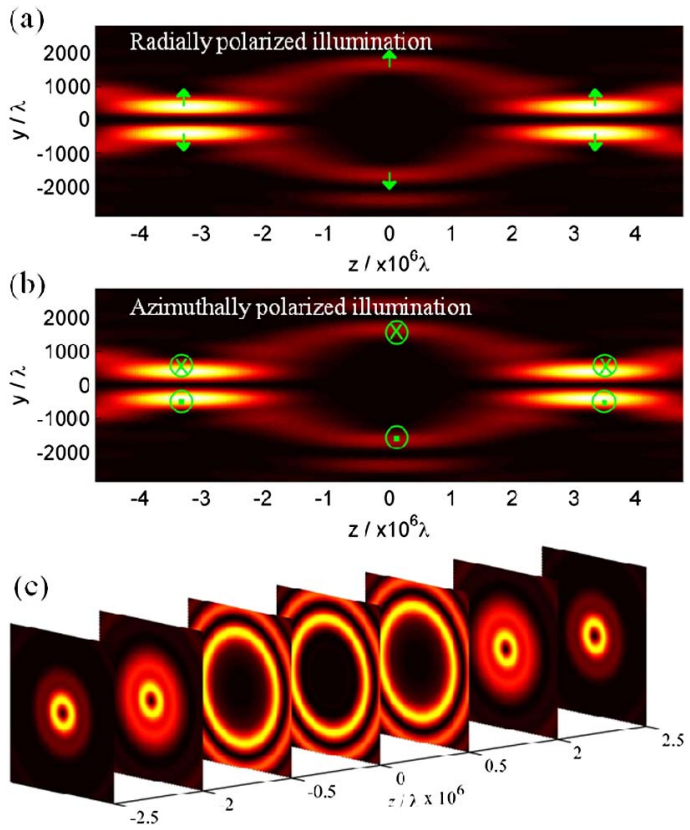

Fig. 3. Electric energy density distributions along the optical axis under incident light with (a) radially and (b) azimuthally polarized fields (where NA $=0.001$ ), and (c) at different positions along the optical axis. The symbols in (a) and (b) denote the polarization directions of the vectorial $\mathrm{BH}$ beam. the electric field intensity distribution in the $x-y$ plane sampled at different positions along the optical axis; if the wavelength of the incident light is $632.8 \mathrm{~nm}$, then the resulting $\mathrm{BH}$ beam has a length of approximately $3.16 \mathrm{~m}$, with a diameter approximately $632 \mu \mathrm{m}$ inside the bottle and $63.2 \mu \mathrm{m}$ at the hollow position. For optical manipulation of microscopic particles, the high-intensity barrier of the hollow or bottle beam may serve as a repelling 'pipe wall' to trap particles in the dark region on the axis while the axial component of thermal force pushes particles along the channel [2,4]. With the $\mathrm{BH}$ beam, for example, particles of diameter $50 \mu \mathrm{m}$ can be transported one by one via this hollow channel and reach the highly volumetric bottle, where dozens of particles can still have good confinement. It is unlike the conventional optical bottle beam, which acts like a highly volumetric container with appropriate confinement, but without the channel to continuously manipulate particles [14]. Moreover, it is also distinct from the optical hollow beam, which cannot be used in manipulating a large amount of particles, even though they are precisely delivered to the desired location. The particles transported to the tail of the hollow will lose control, as the field at the tail of the hollow is diffracted field without good confinement. Hence, the BH beam introduced in this Letter may be applied to manipulate micron-sized lightabsorbing particles over a large distance. In contrast with [13], the bottle and hollow beams are formed together in one single $\mathrm{BH}$ beam combining individual merits of the optical bottle and the hollow beam.

Apart from the NA $=0.001$ example we selected, it should be further stressed that the mask also works for other lenses with different NA choices. To illustrate this, we progressively change the value of NA in the simulation. It is found that both of the length and diameter of
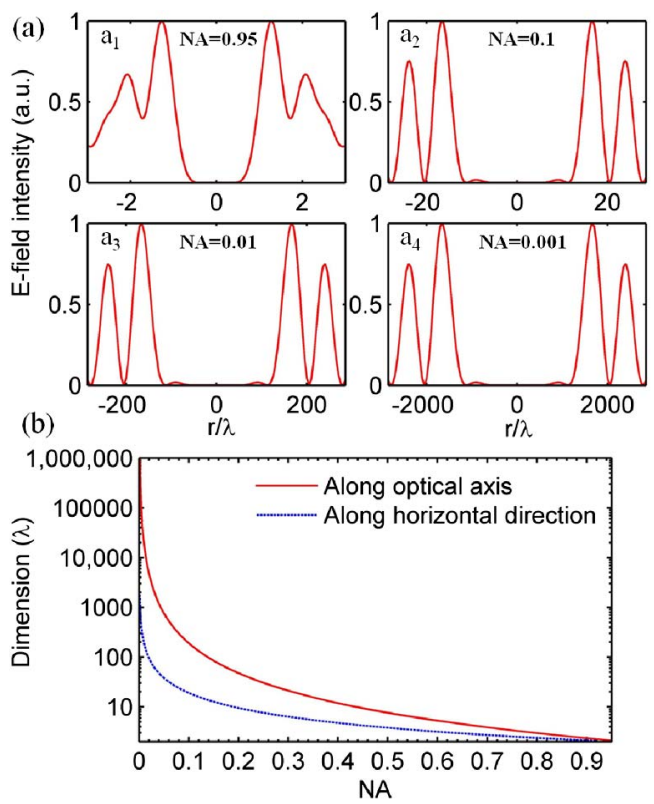

Fig. 4. (a) E-field intensity distribution at the focal plane $(z=0)$ along the radial direction versus different NA. (b) Relationship between $\mathrm{BH}$ beam parameters and NA-red curve denoting $\mathrm{BH}$ beam length (along the optical axis) and blue curve denoting $\mathrm{BH}$ beam radius. 
the $\mathrm{BH}$ beam change according to the NA of the focusing lens. It is evident from the plots presented in Fig. 4(a) that the energy inside the bottle region is null for the high-NA case. Conversely, for the low-NA case, small regions with weak intensity smaller than $2 \%$ appear. However, this will not affect the $\mathrm{BH}$ beam generation due to the negligibly low fraction of energy in these regions. In addition, we observe from Fig. 4(a) that the diameters of the bottle for $\mathrm{NA}=0.95,0.1, \overline{0.01}$, and 0.001 are in the order of $\lambda, 10 \lambda, 100 \lambda$, and $1000 \lambda$, respectively.

Furthermore, the relationship between the length, diameter, and NA can be quantitatively described. We note from Fig. 4(b) that the $\mathrm{BH}$ beam length is inversely proportional to $\mathrm{NA}^{2}$ [i.e., $L \propto \lambda / \mathrm{NA}^{2}$ for the red plot in Fig. 4(b)]; whereas, its diameter is inversely proportional to NA [i.e., $d \propto \lambda /$ NA for the blue plot in Fig. 4(b)]. The advantage of our proposed scheme is that the same binary phase mask may be employed for different choices of NA. Compared with the bottle or hollow beams generated in $[9,10,13]$, what we have proposed is clearly more flexible: the length and diameter of the $\mathrm{BH}$ beam can be conveniently changed by swapping the objectives, thus leading to an adjustable $\mathrm{BH}$ beam. The resulting $\mathrm{BH}$ beam may enhance the flexibility of manipulating light-absorbing particles by using only a single beam [2,4].

In conclusion, we propose a scheme for generating a $\mathrm{BH}$ beam which has an open bottle-shaped region with zero intensity surrounded by a light barrier, and two hollows located at the neck and bottom of this bottle, respectively. For a high-NA lens, the $\mathrm{BH}$ beam can be generated only if the incident illumination is of azimuthal polarization. For a low-NA (NA $\leq 0.1)$ lens, however, the $\mathrm{BH}$ beam can be generated with both radially and azimuthally polarized illumination. It is shown that radially polarized light will lead to $\mathrm{BH}$ beam with radial polarization and azimuthally polarized light will lead to azimuthally polarized $\mathrm{BH}$ beam. In addition, we have found that the binary phase mask does not need to be changed whenever we choose a focusing lens with a different NA; the resulting $\mathrm{BH}$ beam can be controlled to localize at varying positions with different lengths and diameters accordingly ( $\left.L \propto \lambda / \mathrm{NA}^{2}, d \propto \lambda / \mathrm{NA}\right)$, thus leading to an adjustable $\mathrm{BH}$ beam. This $\mathrm{BH}$ beam may find attractive applications in manipulating microsized particles over large distances.

\section{References}

1. V. G. Shvedov, A. V. Rode, Y. V. Izdebskaya, A. S. Desyatnikov, W. Krolikowski, and Y. S. Kivshar, Opt. Express 18, 3137 (2010).

2. P. Zhang, Z. Zhang, J. Prkash, S. Huang, D. Hernandez, M. Salazar, D. N. Christodoulides, and Z. Chen, Opt. Lett. 36, 1491 (2011).

3. Z. Zhang, D. Cannan, J. Liu, P. Zhang, D. N. Christodoulides, and Z. Chen, Opt. Express 20, 16212 (2012).

4. V. G. Shvedov, A. V. Rode, Y. V. Izdebskaya, A. S. Desyatnikov, W. Krolikowski, and Y. S. Kivshar, Phys. Rev. Lett. 105, 118103 (2010).

5. A. S. Desyatnikov, V. G. Shvedov, A. V. Rode, W. Krolikowski, and Y. S. Kivshar, Opt. Express 17, 8201 (2009).

6. A. Ashkin, J. M. Dziedzic, and S. Chu, Opt. Lett. 11, 288 (1986).

7. I. Manek, B. Ovchinnikov, and R. Grimm, Opt. Commun. 147, 67 (1998).

8. Y. Zhang, Opt. Commun. 281, 508 (2008).

9. N. Chattrapiban, E. A. Rogers, D. Cofield, W. T. Hill, and R. Roy, Opt. Lett. 28, 2183 (2003).

10. F. K. Fatemi and M. Bashkansky, Opt. Lett. 31, 864 (2006).

11. A. Kaplan, N. Friedman, and N. Davidson, J. Opt. Soc. Am. B 19, 1233 (2002).

12. V. Pavelyev, V. Osipov, D. Kachalov, S. Khonina, W. Cheng, A. Gaidukeviciute, and B. Chichkov, Appl. Opt. 51, 4215 (2012).

13. M. Wei, W. Shiao, and Y. Lin, Opt. Commun. 248, 7 (2005).

14. J. Arlt and M. J. Padgett, Opt. Lett. 25, 191 (2000).

15. C. Alpmann, M. Esseling, P. Rose, and C. Denz, Appl. Phys. Lett. 100, 111101 (2012).

16. A. H. J. Yang, S. D. Moore, B. S. Schmidt, M. Klug, M. Lipson, and D. Erickson, Nature 457, 71 (2009).

17. H. Wang, L. Shi, B. Lukyanchuk, C. J. R. Sheppard, and C. T. Chong, Nat. Photonics 2, 501 (2008).

18. K. Huang, P. Shi, X. Kang, X. Zhang, and Y. Li, Opt. Lett. 35, 965 (2010).

19. B. Richards and E. Wolf, Proc. R. Soc. London, Ser. A 253, 358 (1959).

20. K. Huang, P. Shi, G. W. Cao, K. Li, X. B. Zhang, and Y. P. Li, Opt. Lett. 36, 888 (2011).

21. R. Dorn, S. Quabis, and G. Leuchs, Phys. Rev. Lett. 91, 233901 (2003).

22. G. Machavariani, Y. Lumer, I. Moshe, A. Mer, and S. Jackel, Opt. Lett. 32, 1468 (2007).

23. N. Jin and Y. Rahmat-Samii, IEEE Trans. Antennas Propag. 55, 556 (2007).

24. K. S. Youngworth and T. G. Brown, Opt. Express 7, 77 (2000). 